\title{
Ueber das Newton'sche Gravitationsgesetz.
}

\section{(Erwiderung).}

In den Astr. Nachr. Nr. 3292 hat Herr Prof. Seeliger eine Entgegnung auf meine »Kritik \& seines Aufsatzes $\gg$ Ueber das Newton'sche Gravitationsgesetz \& veröffentlicht, durch welche ich mich genöthigt sehe, nochmals auf den Gegenstand zurückzukommen. Herr Prof. Seeliger nimmt zunächst die Autorität von Helmholtz für sich in Anspruch. Nach Herrn Prof. Seeliger hat Helmholtz 》vor etwa 20 Jahren ganz allgemeine Folgerungen aus dem Weber'schen Gesetz gezogen, welche mit dem Princip der Erhaltung der Kraft im Widerspruch stehen«. Herr Prof. Seeliger denkt dabei wohl an die Helmholtz'sche Abhandlung über die Theorie der Elektrodynamik (Monatsberichte der Kgl. Preussischen Akademie der Wissenschaften 1872). Helmholtz sagt aber pag. $25 \mathrm{I}$ a. a. O., nachdem er auf die Unmöglichkeit hingewiesen hatte, Kräfte, welche ausser von den Coordinaten noch von den Geschwindigkeiten abhängen, mit dem Princip von der Erhaltung der Kraft in Einklang zu bringen:

"Dagegen diesen noch complicirteren Fall, welchen das Weber'sche Gesetz aufstellt, wo die Kräfte von den Coordinaten und von den ersten und zweiten Differentialquotienten derselben nach der Zeit abhängen, hatte ich damals (Abhandlung über die Erhaltung der Kraft (1 847 ) d. V.) nicht berücksichtigt, und dieser Fall ist mit einer etwas erweiterten Form des Gesetzes von der Erhaltung der Energie allerdings vereinbar.« Herrn Prof. Seeliger dürfte also bei der Niederschrift seiner Entgegnung der Inhalt der betr. Helmholtz'schen Abhandlungen nicht gegenwärtig gewesen sein, da Helmholtz' Ansicht derjenigen, welche ihm von Herrn Prof. Seeliger zugeschrieben wird, gerade entgegengesetzt ist. In der That zeigt Helmholtz, dass, unter Voraussetzung der Gültigkeit des Weber'schen Gesetzes sich unter bestimmten Bedingungen Strömungen entwickeln müssten, welche $\mathrm{zu}$ unendlichen Stromstärken führen. Wie dieses Ergebniss, dessen eigentliche Bedeutung in seinem Widerspruch mit aus anderweitigen Erfahrungen geschöpften physikalischen Anschauungen beruht, zur Entscheidung der vorliegenden Frage beitragen soll, ist für mich nicht ersichtlich.

In seinen weiteren Ausführungen beschränkt sich Herr Seeliger nunmehr auf die Betrachtung des »Unermesslichen im mathematischen Sinne und gelangt in Folge dessen auf dem von ihm eingeschlagenen Wege nur zu sbeliebig grossen, aber endlichen Geschwindigkeiten und Zerrungen «. Damit verschwindet aber überhaupt jeder Widerspruch gegen das Newton'sche Gesetz, denn nach welchem Maassstabe soll die Grenze der im Weltall möglichen oder erlaubten Geschwindigkeiten und Zerrungen festgesetzt werden? Auch hier besitzen wir kein anderes Mittel als die Erfahrung, und dass diese noch recht unvollständig ist, haben gerade in letzter Zeit die Ergebnisse der spectroskopischen Untersuchungen des neuen Sterns im Fuhrmann gezeigt. Da wesentlich neue Gesichtspunkte durch die Erwiderung des Herrn Prof. Seeliger nicht gewonnen sind, so würde mir ein weiteres Eingehen auf den Gegenstand nicht erforderlich scheinen, wenn mir Herr Prof. Seeliger nicht in den noch folgenden Sätzen einige Vorwürfe gemacht hätte, die ich zurückzuweisen genöthigt bin.

Wenn ich sagte, dass die Widersprüche, zu welchen die Vorstellung des unendlichen Weltalls als einer Kugel, im vorliegenden Falle führen, nur beweisen, dass die Formen mathematischer Untersuchung versagen, sobald man den Boden des Vorstellbaren verlässt und diese Formen auf ein transcendentales Problem anwendet, so enthalten diese Sätze nur denselben Gedanken, welchem Riemann im Eingang des letzten Abschnitts seiner Habilitationsschrift »Ueber die Hypothesen, welche der Geometrie zu Grunde liegen « einen präciseren Ausdruck verleiht mit den Worten: „Die Fragen über das Unmessbargrosse sind für die Naturerklärung müssige Fragen.s Wenn Herr Prof. Seeliger weiter fragt, woher meine Kenntniss des Satzes stamme, dass die Wirkung des Newton'schen Gesetzes die Zerstörung der gedachten Massenvertheilungen zur Folge haben würde und ihre Ersetzung durch solche Vertheilungen, welche eben mit inm verträglich sind, so brauche ich nur auf die „Principia « hinzuweisen, da der Satz nichts weiter sagt, als die "Leges Motus «, welche der Lehre von der Mechanik zur Grundlage dienen. Mit seiner Verwahrung gegen die Hypothese einer zeitlichen Ausbreitung der Anziehungskraft bekämpft Herr Prof. Seeliger einen nicht vorhandenen Gegner, da eine derartige Behauptung nirgends von mir aufgestellt ist. Ebensowenig habe ich »ziemlich unverständlich « von der Verwandlung eines »dynamischen Systems in ein statisches « gesprochen, sondern von der Verwandlung eines dynamischen Problems in ein statisches.

Auf die von Herrn Prof. Seeliger aufgestellten Integrale würde ich nicht noch einmal zurückkommen, wenn derselbe nicht die Richtigkeit seiner Darstellung mit einiger Lebhaftigkeit vertheidigte. Herr Prof. Seeliger sagt in seinem ersten Aufsatz: »Andererseits sind die oben erwähnten Schwierigkeiten vollkommen verschwunden, denn die Integrale :

$$
\int_{R_{0}}^{R_{1}} \delta e^{-\lambda r} r \mathrm{~d} r, \quad \int_{R_{0}}^{R_{1}} \delta e^{-\lambda r} \mathrm{~d} r, \int_{R_{0}}^{R_{1}} \delta e^{-\lambda r} \frac{\mathrm{d} r}{r}
$$

haben endliche Werthe. \& Diese Ausdrucksweise führt zunächst zu der unzutreffenden Vorstellung, dass die vorstehenden Integrale bei dem neuen Anziehungsgesetz unmittelbar an die Stelle der für das Newton'sche Gesetz gültigen Integrale treten. Jedenfalls kann man sich aber von der Richtigkeit der Behauptung des Herrn Prof. Seeliger nur überzeugen, wenn man die strengen Ausdrücke, nicht aber bereits näherungsweise ausgewerthete Formen vor sich hat, wie das von Herrn. Prof. Seeliger nunmehr hinzugefügte Integral :

$$
-\varepsilon \int_{R_{0}}^{R_{1}} \delta e^{-\lambda r} r \mathrm{~d} r
$$


Mit gleichem Recht hätte die Mittheilung des ersten der oben stehenden drei Integrale

$$
\int_{R_{0}}^{R_{1}} \delta e^{-\lambda r} \mathrm{~d} r
$$

genügt, da durch die Endlichkeit desselben die Endlichkeit der übrigen gleichfalls »vollständig « charakterisirt ist, wie man sofort sieht, wenn man für $R_{0}$ und $R_{1}$ die Werthe I resp. $\infty$ einsetzt.

Ich schliesse mit den der oben angeführten Abhand-

Potsdam, Königl. Observatorium, 1895 Juli. lung Riemann's entnommenen Sätzen, welche sich auch auf den vorliegenden Gegenstand beziehen lassen :

"Die Entscheidung dieser Fragen kann nur gefunden werden, indem man von der bisherigen durch die Erfahrung bewährten Auffassung der Erscheinungen, wozu Newton den Grund gelegt, ausgeht und diese durch Thatsachen, die sich aus ihr nicht erklären lassen, getrieben allmählich umarbeitet; solche Untersuchungen, welche, wie die hier gefuhrte, von allgemeinen Begriffen ausgehen, können nur dazu dienen, dass diese Arbeit nicht durch die Beschränktheit der Begriffe gehindert und der Fortschritt im Erkennen des $\mathrm{Zu}$ sammenhangs der Dinge nicht durch überlieferte Vorurtheile gehemmt wird.

Dr. Wilsing.

\section{Bemerkung zur vorstehenden Erwiderung.}

Auf die erste Notiz des Herrn Dr. Wilsing habe ich nur geantwortet, weil ich glaubte, einige aufklärende Worte würden genügen, Herrn Dr. Wilsing die Ueberzeugung zu verschaffen, dass er nicht genügend überlegte Meinungen etwas übereilt der Oeffentlichkeit übergeben habe. Die vorstehende Erwiderung liefert nun freilich den Beweis, dass ich mich in dieser Beziehung getäuscht habe und dass Herr Dr. Wilsing seine unbegründeten Einwände aufrecht erhält. Ich kann selbstverständlich hierin keine Veranlassung finden, die Sachlage von Neuem zu beleuchten, vielmehr glaube ich, dass sich Jeder, der sich für die besprochene Frage interessirt, leicht selbst ein Urtheil über die Berechtigung der Einwände des Herrn Dr. Wilsing bilden wird. Im Uebrigen werde ich das von mir aufgestellte Problem in kurzer Zeit weiter bearbeiten, ganz unbeirrt von den belanglosen Bemerkungen des Herrn Dr. Wilsing. Ich halte eine Discussion auf Grund der letzteren für ganz unnöthig, werde dieselbe unter keinen Umständen weiter führen und betrachte den Gegenstand in dieser Richtung als vollständig erledigt.

Auf das Entschiedenste muss ich aber gegen die Art protestiren, wie Herr Dr. Wilsing Aussprijche von Helmholtz, Riemann, Newton citirt und solche aus dem Zusammenhang gerissene Citate verwerthet. Auf die Citate aus Riemann's Arbeiten will ich ebenso wenig eingehen, wie auf die Thatsache, dass Herr Dr. Wilsing eine nachweisbar ganz falsche mechanische Vorstellung auf die Autorität Newton's zurückzuführen sucht. Denn die Aeusserungen Riemann's passen, wie leicht zu sehen, gar nicht auf das vorliegende Problem und die Berufung auf Newton's Principien kann nur aus einem Missverständnisse der mechanischen Grundsätze hervorgegangen sein. Ueber die Sicherheit aber, mit welcher Herr Dr. Wilsing Helmholtz'sche Sätze heranzieht, ohne eine genügende Kenntniss der in Frage kommenden Originalarbeiten zu haben, wird sich jeder Sachverständige höchlichst verwundern müssen. Nicht unberechtigt dürfte doch wohl die Erwartung sein, dass derjenige, welcher eine Polemik beginnt, in dem betreffenden Gebiete wenigstens beiläufig orientirt sei. Herr Dr. Wilsing scheint aber nur die als »vorläufige Anzeige» der bekannten grossen Arbeit von Helmholtz (Crelle's Journal Bd. 75) in den Monatsberichten der Berliner Akademie abgedruckte Mittheilung zu kennen.
Hätte er die erwähnte Abhandlung und die grundlegende frühere (Crelle Band 72) angesehen, so hätte er vielleicht selbst erkannt, dass er sich durch einen falsch verstandenen Satz hat irreführen lassen. Die Sache verhält sich nicht wie Herr Dr. Wilsing sie darstellt, sondern folgendermaassen. Jedes Kraftgesetz genügt dem Satze von der lebendigen Kraft, wenn es eine Kräftefunction hat. Dieses trifft beim Weber'schen Gesetz zu, trotzdem widerspricht es dem Princip der Erhaltung der Kraft in dem Sinne, dass es die Möglichkeit zulässt, aus endlicher Geschwindigkeit in endlicher Entfernung unendlich grosse Arbeitsleistung zu gewinnen. Das ist eben die Helmholtz'sche Entdeckung gewesen, die seiner Zeit berechtigtes Aufsehen gemacht hat. Eine Discussion über diese allbekannte Thatsache muss ich als ganz unzulässig zurückweisen. Um aber auch dem Fernerstehenden unzweifelhaft die Unrichtigkeit der Auffassung des Herrn Dr. Wilsing zu zeigen, wird es genügen, folgenden Satz wörtlich aus der ersten Abhandlung von Helmholtz anzufuhren (Wissenschaftl. Abhandlungen I p. 553): „Dieses (Weber's) Gesetz fügt sich allerdings insofern dem Gesetz von der Erhaltung der Kraft ein, als es keinen $\mathrm{Kr}$ eisprocess zulässt, der Arbeit aus Nichts erzeugte. Aber es widerspricht insofern, als zwei elektrische Theilchen, die sich nach diesem Gesetz bewegen und mit endlicher Geschwindigkeit beginnen, in endlicher Entfernung von einander unendliche lebendige $\mathrm{Kraft}$ erreichen und also eine unendlich grosse Arbeit leisten können. *

Aber auch die Stellung, welche Helmholtz zu den von mir benutzten Grundsätzen einnimmt, findet in überaus grosser Deutlichkeit, die ebenfalls jede weitere Discussion abschneidet, Aussprache und zwar in der zweiten Abhand. lung (a. a. O.I p. 658 ):

"Wenn wir aber das Weber'sche Gesetz als ein wirklich elementares anerkennen wollen, als ein solches, welches den letzten Grund der betreffenden Erscheinungen vollständig ausspricht und nicht nur als einen angenähert richtigen Ausdruck der Thatsachen für gewisse engere Grenzen, so müssen wir verlangen, dass es, auch auf Objecte von den denkbar grössten Dimensionen angewendet, Folgerungen gebe, die physikalisch möglich sind. \&

Dieser Satz ist nicht etwa eine beiläufige Bemerkung, 